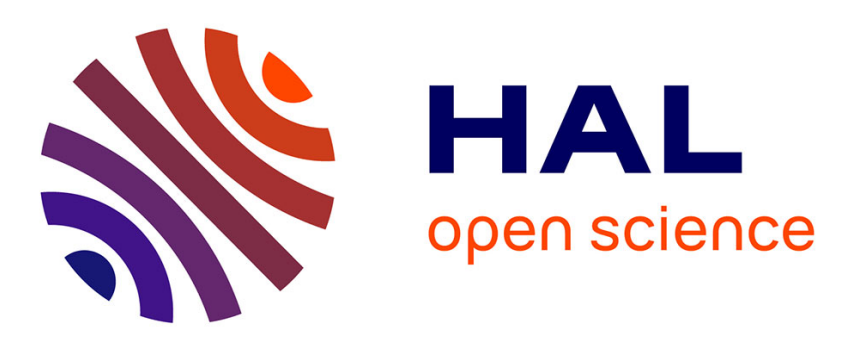

\title{
MRF agent based segmentation: Application to MRI brain scans
}

\author{
Benoit Scherrer, Dojat Michel, Florence Forbes, Catherine Garbay
}

\section{To cite this version:}

Benoit Scherrer, Dojat Michel, Florence Forbes, Catherine Garbay. MRF agent based segmentation: Application to MRI brain scans. AIME 2007 - 11th Conference on Artificial Intelligence in Medicine, Jul 2007, Amsterdam, Netherlands. pp.13-23, 10.1007/978-3-540-73599-1_2 . inserm-00519836

\section{HAL Id: inserm-00519836 https://www.hal.inserm.fr/inserm-00519836}

Submitted on 8 Jun 2011

HAL is a multi-disciplinary open access archive for the deposit and dissemination of scientific research documents, whether they are published or not. The documents may come from teaching and research institutions in France or abroad, or from public or private research centers.
L'archive ouverte pluridisciplinaire HAL, est destinée au dépôt et à la diffusion de documents scientifiques de niveau recherche, publiés ou non, émanant des établissements d'enseignement et de recherche français ou étrangers, des laboratoires publics ou privés. 


\title{
MRF Agent Based Segmentation: Application to MRI Brain Scans.
}

\author{
anonymous \\ 1 anonymous, \\ 2 anonymous, \\ 3 anonymous
}

\begin{abstract}
The Markov Random Field (MRF) probabilistic framework is classically introduced for a robust segmentation of Magnetic Resonance Imaging (MRI) brain scans. Most MRF approaches handle tissues segmentation via global model estimation. Structure segmentation is then carried out as a separate task. We propose in this paper to consider MRF segmentation of tissues and structures as two local and cooperative procedures immersed in a multiagent framework. Tissue segmentation is performed by partitionning the volume in subvolumes where agents estimate local MRF models in cooperation with their neighbours to ensure consistency of local models. These models better reflect local intensity distributions. Structure segmentation is performed via dynamically localized agents that integrate anatomical spatial constraints provided by an a priori fuzzy description of brain anatomy. Structure segmentation is not reduced to a postprocessing step: rather, structure agents cooperate with tissue agents to render models gradually more accurate. We report several experiments that illustrate the working of our multiagent framework. The evaluation was performed using both phantoms and real $3 \mathrm{~T}$ brain scans and showed a robustness to nonuniformity and noise together with a low computational time. This MRF agent based approach appears as a very promising new tool for complex image segmentation.
\end{abstract}

\section{Introduction}

MRI Brain Scan Segmentation is a challenging task and has been widely addressed in the last 15 years. Difficulties arise from various sources including the size of the data, the high level of noise with strong field images (3T or higher), the intensity nonuniformity or the low contrast between tissues. The Markov Random Field (MRF) probabilistic framework is classically used to introduce spatial dependencies between voxels, providing a labeling regularization and a robust to noise segmentation [1],[2]. In addition to noise, the intensity nonuniformity is a critical issue. Most approaches estimate global tissue intensity models through the entire volume and require the estimation of an explicit "bias field" model to account for the intensity nonuniformity [3],[4]. These models are based on underlying assumptions that are not always valid and requires additional computational burden for their estimation. Local segmentation approaches are 
attractive alternatives [5],[6],[7]. The principle is to locally compute the tissue intensity models in various volume partitions. These models fit better to local image properties. In particular local segmentation intrinsically handles the intensity nonuniformity without any bias field modelization. Existing approaches either use local estimation as a preprocessing step to estimate a bias field model [5], or use redondant information to ensure consistency and smoothnesss between local estimated models [7], greedily increasing computation. We consider that these approaches do not fully take advantage of locality. We propose to immerse a local MRF segmentation approach into a distributed and cooperative framework based on the multiagent (MA) paradigm. This paradigm provides convenient mechanisms of cooperation and coordination, allowing to ensure consistency of local models in an elegant way. In addition, we achieve segmentation of tissues and structures in a unified and cooperative way. We show how to design a MRF agent-based segmentation approach and report some experiments on MRI brain scans that exhibit the properties of the system. These efforts extend a first tentative of agent based region growing tissue segmentation [6] to a unified MRF tissue and structure segmentation.

This paper is organized as follows. We present in Section 2 how MRF processes are turned into cooperating agent entities. Section 3 reports evaluation results and presents several experiments to exhibit some interesting properties of such a local approach. Section 4 is devoted to the discussion and the conclusion.

\section{MRF Agent Based Segmentation}

\subsection{MRF Framework}

We consider a finite set of $\mathrm{N}$ voxels $V=\{1, \ldots N\}$ on a regular three-dimensional (3-D) grid. Our aim is to assign each voxel $i$ to one of $K$ classes considering the observed greylevel intensity $y_{i}$ at voxel $i$. Both observed intensities and unknown classes are considered to be random fields denoted respectively by $\mathbf{Y}=\left\{Y_{1}, \ldots, Y_{N}\right\}$ and $\mathbf{Z}=\left\{Z_{1}, \ldots, Z_{N}\right\}$. Each random variable $Z_{i}$ takes its value in $\left\{e_{1}, \ldots, e_{K}\right\}$ where $e_{k}$ is a $K$-dimensional binary vector corresponding to class $k$. Only the $k^{t h}$ component of this vector is non zero and is set to 1 . In a traditionnal Markov model based segmentation framework, it is assumed that the conditional field $\mathbf{Z}$ given $\mathbf{Y}=\mathbf{y}$ is a Markov random field, ie.

$$
p(\mathbf{z} \mid \mathbf{Y}=\mathbf{y}, \boldsymbol{\Phi})=W_{y, \Phi}^{-1} \exp (-H(\mathbf{z} \mid \mathbf{y}, \Phi))
$$

where $H(\mathbf{z} \mid \mathbf{y}, \Phi)$ is an energy function depending on some parameters $\Phi=$ $\left(\Phi_{y}, \Phi_{z}\right)$ and given by:

$$
H(\mathbf{z} \mid \mathbf{y}, \Phi)=H\left(\mathbf{z} \mid \Phi_{z}\right)-\sum_{i \in V} \log p\left(y_{i} \mid z_{i}, \Phi_{y}\right) .
$$

This energy is a combination of two terms: the first term in (1) is a regularization term that accounts for spatial dependencies between voxels. Denoting by $\mathcal{N}(i)$ 
the neighbors of voxel $i$, we will consider a Potts model with external field:

$$
H\left(\mathbf{z} \mid \Phi_{z}\right)=\sum_{i \in V}\left[{ }^{t} z_{i} v_{i}-\frac{\beta}{2} \sum_{j \in \mathcal{N}(i)}{ }^{t} z_{i} z_{j}\right]
$$

The second summation above tends to favor neighbors that are in the same class when parameter $\beta$ is positive. This $\beta$ parameter accounts for the strengh of spatial interaction. Other parameters are the $v_{i}$ 's that are K-dimensional vectors defining the so-called external field. In this case $\Phi_{z}=\left\{v_{1}, \ldots, v_{N}, \beta\right\}$. The $v_{i}$ 's can be related to a priori weights accounting for the relative importance of the $K$ classes at site $i$. The introduction of these extra parameters in the standard Potts model enables us to integrate a priori knowledge on classes. The second term in (1) is a data-driven term based on intensities. For MRI we generally consider a Gaussian probability density function for the observed intensity $y_{i}$ when the tissue class is $z_{i}$. It follows that $p\left(y_{i} \mid z_{i}=e_{k}, \Phi_{y}\right)=g_{\mu_{k}, \sigma_{k}}\left(y_{i}\right)$ with $\Phi_{y}=\left\{\mu_{k}, \sigma_{k}, k=1 \ldots K\right\}$. Segmentation is then performed according to the Maximum A Posteriori principle (MAP) by maximizing over $\mathbf{z}$ the probability $p(\mathbf{z} \mid \mathbf{y}, \Phi)$. This requires the evaluation of an intractable normalizing constant $W_{\mathbf{y}, \Phi}$ and the estimation of the unknown parameters $\Phi$. A standard approach is to use the ICM algorithm that alternates between parameter estimation and segmentation but results in biased estimates. EM-based algorithms and variants proposed by [8] can be rather considered. They are based on Mean-field like approximations which make the MRF models case tractable. In all these approaches, MRF estimation is performed globally through the entire volume.

\subsection{Local Approach of Tissue Segmentation}

We agentify the global MRF process by distributing in the volume several local MRF processes in a MA paradigm.

We consider a decentralized and memory shared MA framework based on the classical Agent/Group/Behaviour conceptual model. At the system starting point, a global tissue agent $\mathcal{A}_{G}^{T}$ is responsible for partitioning the volume into $C$ nonoverlapping territories $\left\{\mathcal{T}_{c}^{T}, c=1 \ldots C\right\}$ and instantiating one situated tissue agent $\mathcal{A}_{c}^{T}$ per territory. We consider $K=3$ tissue classes: $C S F$ (Cephalo-Spinal Fluid), GM (Grey Matter) and $W M$ (White Matter). The hidden tissue classes $t_{i}$ 's take their values in $\left\{e_{1}, e_{2}, e_{3}\right\}$ respectively for classes $\left\{e_{C S F}, e_{G M}, e_{W M}\right\}$. The tissue agent $\mathcal{A}_{c}^{T}$ segments its territory with the MRF model defined by the energy (see Equations (1) and (2)):

$$
H^{c}\left(\mathbf{t} \mid \mathbf{y}, \Phi^{c}\right)=\sum_{i \in \mathcal{T}_{c}^{T}}\left[{ }^{t} t_{i} \lambda_{i}^{c}-\frac{\beta^{c}}{2} \sum_{j \in \mathcal{N}(i)}{ }^{t} t_{i} t_{j}-\log p\left(y_{i} \mid t_{i}, \Phi_{y}^{c}\right)\right]
$$

where the parameters $\Phi^{c}=\left\{\Phi_{t}^{c}, \Phi_{y}^{c}\right\}$ have to be estimated. The external field denoted by $\left\{\lambda_{i}^{c}, i \in \mathcal{T}_{c}^{T}\right\}$ is not estimated but used to incorporate information 
coming from structure segmentation agents (see Section 2.3). Each tissue agent owns three behaviours to segment its territory: 1) Tissue_Init which initializes the EM algorithm by computing initial intensity models via a Fuzzy-C Mean algorithm (FCM), 2) Tissue_EM which computes the MRF model parameters in cooperation with neighbouring agents and 3) Tissue_Stabilized when the agent is in the idle state.

Cooperation between tissue agents: The agent $\mathcal{A}_{c}^{T}$ cooperates with its neighbors group denoted by $\mathcal{G}_{N}\left(\mathcal{A}_{c}^{T}\right)$ to ensure a global consistency of the local estimated model. It performs:

Model Checking: for each tissue class $k$ we compute a local mean model from the models of $\mathcal{G}_{N}\left(\mathcal{A}_{c}^{T}\right)$. The KullBack-Leibler distance $\mathcal{D}_{k}^{c}$ provides a measure of dissimilarity between intensity models of each class that allows to apply model correction if required.

Model Correction: we compute the corrected mean and variance of class $k$ from a linear combination of estimated and mean models according to $\mathcal{D}_{k}^{c}$.

Model Interpolation: we use cubic splines interpolation between corrected models of $\mathcal{A}_{c}^{T}$ and of $\mathcal{G}_{N}\left(\mathcal{A}_{c}^{T}\right)$ to compute one intensity model $\left\{g_{\mu_{i, k}^{c}, \sigma_{i, k}^{c}}, i \in \mathcal{T}_{c}^{T}, k \in\right.$ $\left.\left\{e_{C S F}, e_{G M}, e_{W M}\right\}\right\}$ per voxel. There are two benefits from interpolating models. First, this ensures smooth model variation between neighboring agents. Second, this intrinsically handles nonuniformity of intensity inside each agent.

Coordination between tissue agents: Each agent enter in idle mode after its initialization. The global tissue agent $\mathcal{A}_{G}^{T}$ then computes a global tissue intensity model using the FCM algorithm and wakes up the 20 percents agents whose local model is nearest from the global model. Once each $\mathcal{A}_{c}^{T}$ has finished its local model estimation, it wakes up its neighbors. First, it allows them to perform estimation in turn. Second, it allows already stabilized agent to perform model checking, restarting their estimation if needed to take into account updated models of $\mathcal{A}_{c}^{T}$ in their model correction and model interpolation.

\subsection{Cooperative Approach of Tissue and Structure Segmentation}

Automatic structures segmentation can not rely only on radiometry information because intensity distributions of grey nucleus are largely overlapping. A priori knowledge should be introduced. Classical approaches rely on an a priori known atlas describing anatomical structures. Atlas warping methods are however time consuming and limited due to inter-subject variability. A recent different way to introduce a priori anatomical knowledge is to describe brain anatomy with generic fuzzy spatial relations [9],[10]. We generally consider three kind of spatial relations: distance, symmetry and orientation relations. They are expressed as $3 \mathrm{D}$ fuzzy maps to take into account the general nature of the provided knowledge. We describe each subcortical structure by a set of generic fuzzy spatial relations provided by a brain anatomist. Fusion operators between fuzzy sets then permits to combine the knowledge provided by each spatial relation and provides a Fuzzy Localization Map (FLM) of the structure in the volume.

We define one structure agent $\mathcal{A}_{l}^{S}$ per structure and currently consider $L=9$ subcortical structures: the ventricular System, the two Frontal Horns, the two 
Caudate Nucleus, the two Thalamus, and the two Putamens. Each structure agent segments its territory with a MRF model of $K=2$ classes referred as structure and background. The FLM $f^{l}$ of structure $l$ is used in two ways: first it dynamically provides the structure territory $\mathcal{T}_{l}^{S}$ containing the structure $l$ by a simple thresholding. Second we propose to integrate it as an a priori anatomical knowledge in the MRF framework. Denoting by $\mathbf{s}=\left\{s_{i}, i \in \mathcal{T}_{l}^{S}\right\}$ the hidden classes, the energy function of the MRF model of $\mathcal{A}_{l}^{S}$ is given by:

$$
H^{l}\left(\mathbf{s} \mid \mathbf{y}, \Psi^{l}\right)=\sum_{i \in \mathcal{T}_{l}^{S}}\left[{ }^{t} s_{i} \alpha_{i}^{l}-\frac{\beta^{l}}{2} \sum_{j \in \mathcal{N}(i)}{ }^{t} s_{i} s_{j}-\log p\left(y_{i} \mid s_{i}, \Psi_{y}^{l}\right)\right],
$$

with $\Psi^{l}=\left\{\Psi_{s}^{l}, \Psi_{y}^{l}\right\}$ and $s_{i} \in\left\{e_{1}, e_{2}\right\}=\left\{e_{B}, e_{S}\right\}$ for a voxel of the background or a voxel belonging to structure $l$. The external field denoted by $\left\{\alpha_{i}, i \in \mathcal{T}_{l}^{S}\right\}$, where $\alpha_{i}^{l}$ is a 2-dimensional vector, allows to incorporate the a priori knowledge contained in the FLM. We denote by $f_{i}^{l}$ the value of $f^{l}$ at voxel $i$ and propose to introduce the prior fuzzy knowledge of the FLM as relative prior weights for each voxel $i$, by setting:

$$
\alpha_{i}^{l}=\gamma\left[\begin{array}{c}
-\log \left(1-f_{i}^{l}\right) \\
-\log f_{i}^{l}
\end{array}\right]
$$

where $\gamma$ adjusts the influence of the external field. When $f_{i}^{l} \approx 0$, the voxel $i$ is unlikely to belong to the structure. If $\gamma$ was null, the segmentation would be performed only from the intensity models. Else, according to $(5), \alpha_{i}^{l}(1)<\alpha_{i}^{l}(2)$ which favores in (4) the class background. When $f_{i}^{l} \approx 1$, the voxel $i$ is likely to belong to the structure. In that case $\alpha_{i}^{l}(1)>\alpha_{i}^{l}(2)$ and the class structure is favored.

Each structure owns four behaviours: 1) Struct_Init that initializes the structure agent, 2) Struct_ComputeFuzzyMap that computes or updates the FLM from fuzzy spatial relations, 3) Struct_EM that computes MRF model parameters and 4) Struct_Stabilized when the agent is in the idle state. As we define a neighborhood group for each tissue agent, we define the group $\mathcal{G}_{T \rightarrow S}\left(\mathcal{A}_{l}^{S}\right)$ of tissue agents cooperating with a structure agent $\mathcal{A}_{l}^{S}$, the group $\mathcal{G}_{S \rightarrow T}\left(\mathcal{A}_{c}^{T}\right)$ of structure agents cooperating with a tissue agent $\mathcal{A}_{c}^{T}$, and the group $\mathcal{G}_{S \rightarrow S}\left(\mathcal{A}_{l}^{S}\right)$ of structure agents using $\mathcal{A}_{l}^{S}$ as a reference in a spatial relation. These groups allow to detail cooperation and coordination mechanisms between agents.

Updating structure models via tissue models: each structure being composed of a single tissue $T^{l} \in\left\{e_{C S F}, e_{G M}, e_{W M}\right\}$, we do not estimate intensity model $\Psi_{y}^{l}$ of class structure and class background. We rather compute them from tissue intensity models of $\mathcal{G}_{T \rightarrow S}\left(\mathcal{A}_{l}^{S}\right)$ estimated by tissue agents, by setting:

$$
\left\{\begin{array}{l|l}
p\left(y_{i}\right. & \left.s_{i}=e_{S}, \Psi_{y}^{l}\right)=p\left(y_{i} \mid t_{i}=T^{l}, \Psi_{y}\right) \\
p\left(y_{i}\right. & \left.s_{i}=e_{B}, \Psi_{y}^{l}\right)=\underset{t \in\left\{e_{C S F}, e_{G M}, e_{W M}\right\}}{\max } p\left(y_{i} \mid t_{i}=t, \Psi_{y}\right),
\end{array}\right.
$$

so that improvements in tissue intensity models estimation will be dynamically taken into account by structure agents. 
Feedback of Structure Segmentation on Tissue Segmentation: conversely, results from structure agents are integrated in the tissue segmentation model via the external field $\lambda^{c}$ of tissue agents. We express it as the disjunctive fusion of a posteriori probabilities $p\left(\mathbf{s} \mid \mathbf{y}, \Psi^{l}\right)$ coming from structures of $\mathcal{G}_{S \rightarrow T}\left(\mathcal{A}_{c}^{T}\right)$. It follows that structure segmentation is not reduced to a second step but is combined with tissue segmentation to improve its performance.

Update Fuzzy Maps: when the segmentation of structure $l$ is updated the structure models of $\mathcal{G}_{S \rightarrow S}\left(\mathcal{A}_{l}^{S}\right)$ take it into account by re-computing their spatial relations with respect to $l$, making the knowledge gradually more accurate.

\section{Evaluation}

The evaluation was performed using both phantoms and real 3T brain scans. We first evaluated the tissue segmentation performances provided by tissue agents only. We quantitatively compared our approach to two well known ap-

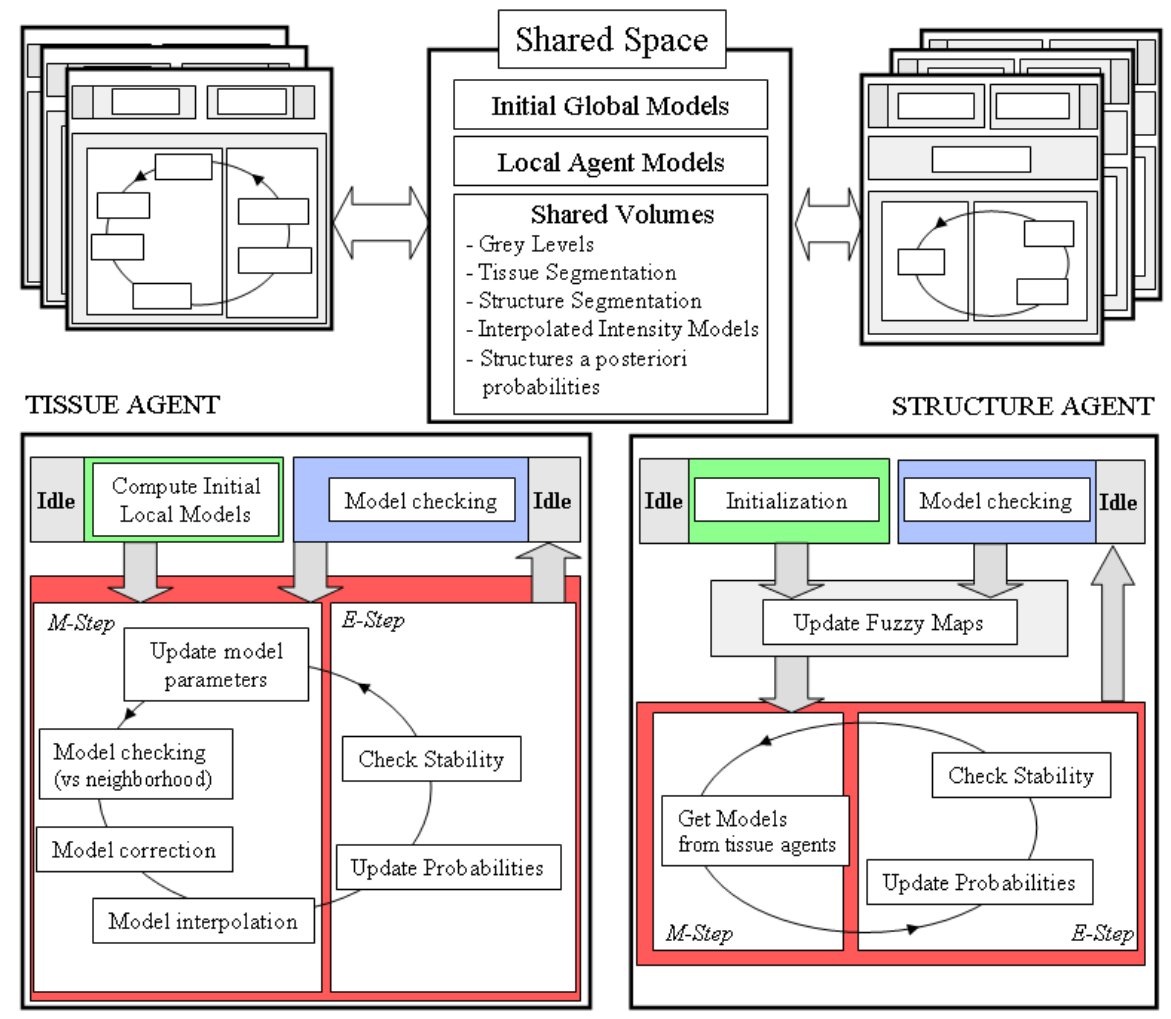

Fig. 1. Synthetic view of our agent based approach. 


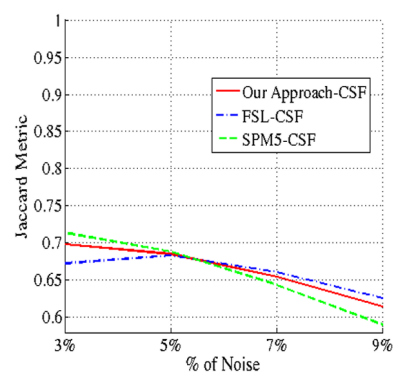

(a)

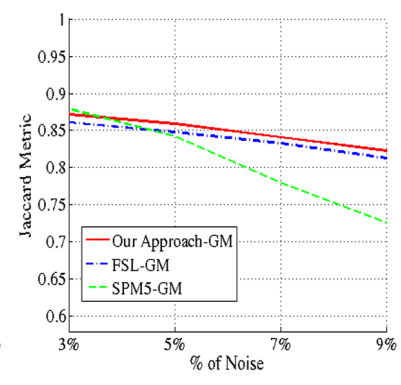

(b)

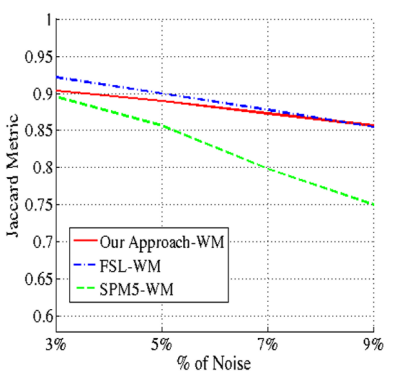

(c)

Fig. 2. Comparison of our approach to FSL and SPM5 for tissue segmentation on the BrainWeb phantoms with $40 \%$ of nonuniformity and different noise values. Evaluation for class CSF (a), GM (b) and WM (c) classes.

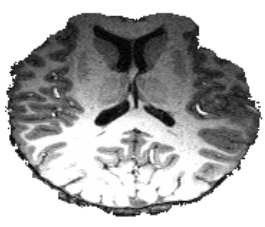

(a)

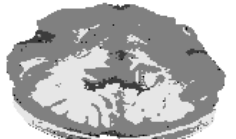

(b)

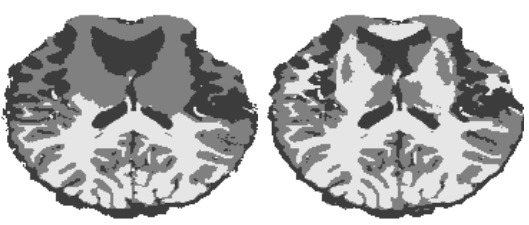

(c)

(d)

Fig. 3. Evaluation on a MRI brain scan with very high intensity nonunformity (a). Tissue segmentation provided by SPM5 (b), FSL (c) and our approach (d).

proaches, FSL [2] and SPM5 [4], with the Jaccard similarity ${ }^{4}$ on the BrainWeb ${ }^{5}$ [11] database (see Figure 2). It shows comparable results and particularly more robustness to noise than SPM5, whereas the computational time was approximately $4 \mathrm{~min}$ with our approach and respectively $8 \mathrm{~min}$ and $14 \mathrm{~min}$ with FSL and SPM5 on a $4 \mathrm{Ghz}$ Pentium, 1Go RAM. The Figure 3 shows visual evaluation on a very high bias field brain scan ${ }^{6}$. SPM5, which uses an a priori atlas, failed in the segmentation while FSL did not estimate a correct bias field. Our local approach clearly appears to be more robust to very high intensity inhomogeneities. Figure 4 shows results of cooperative tissue and structure segmentation. In addition to these competitive performances, our platform allows to exhibit some interesting properties of this local approach. Figure 5 shows that large territory sizes result

\footnotetext{
${ }^{4}$ The Jaccard similarity between two sets is defined by the size of their intersection divided by the size of their union. A value of 1.0 represents a complete agreement.

${ }^{5}$ BrainWeb provides MR volumes whose the segmentation is known (semi-manually segmented by experts) and to which different values of noise and nonuniformity can be added.

6 This image was acquired with a surface coil which provides a high sensitivity in a small region (here the occipital lobe) and is useful for functional imaging.
} 


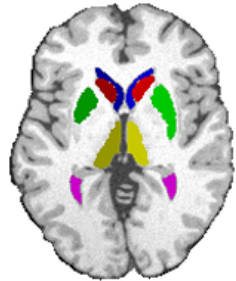

(a)

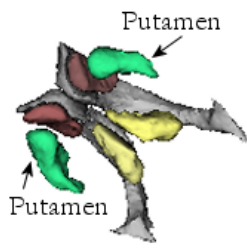

(b)

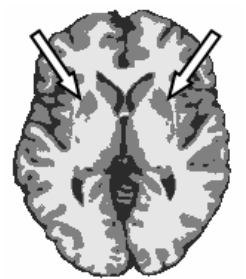

(c)

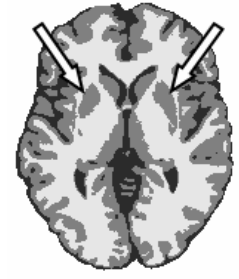

(d)

Fig. 4. Cooperative tissue and structure segmentation: structure segmentation (a) with $3 \mathrm{D}$ rendering (b) shows good results with computational time less than 15 minutes. Image (d) shows visual improvement in tissue segmentation compared to the segmentation produced by tissue agents only (c) (see putamens).

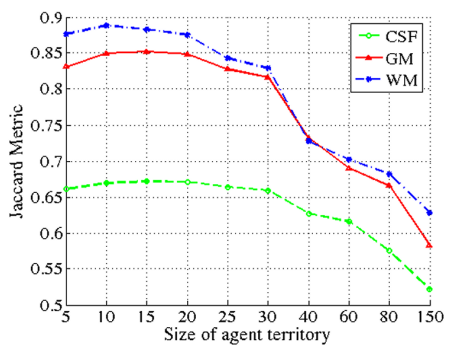

\begin{tabular}{cc}
\hline \multicolumn{3}{c}{$\begin{array}{c}\text { Size of agent } \\
\text { territory }\end{array}$} & $\begin{array}{c}\text { Nubents } \\
\text { agents }\end{array}$ \\
\hline 5 & 17422 \\
10 & 2474 \\
15 & 807 \\
20 & 379 \\
25 & 212 \\
30 & 137 \\
40 & 76 \\
60 & 18 \\
80 & 8 \\
150 & 1 \\
\hline
\end{tabular}

Fig. 5. Influence of tissue agent territory size on the robustness to intensity nonuniformity, using the BrainWeb phantom with $3 \%$ of noise and $100 \%$ of nonuniformity.

in poor performance. Smaller territory sizes allow to better model local intensity distributions, but need to be large enough to correctly estimate models. In practice we use territory size of 20x20x20 voxels. Figure 6 illustrates the activity of a tissue agent immersed in the system. It allows to understand the behaviour of an agent that would have poor estimated models without cooperation mechanisms.

\section{Discussion and Conclusion}

We propose an original MRF agent based approach for MRI brain scan segmentation. Markovian segmentation is an efficient probabilistic framework which introduces a local regularization via spatial dependancies between voxels. It makes it robust to local pertubations such as data noise. In addition, accurate segmentation of MRI brain scan requires to handle the intensity nonuniformity. These global perturbations require to spatially adapt models over the volume. Instead of estimating a spatial bias field model or using non-tractable non-stationnary MRFs, we propose an original agent based approach: we integrate the local level of regularization via Markov models and the global level via distributed 


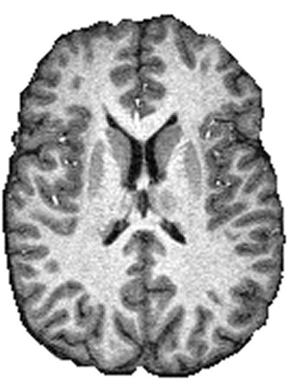

(a)

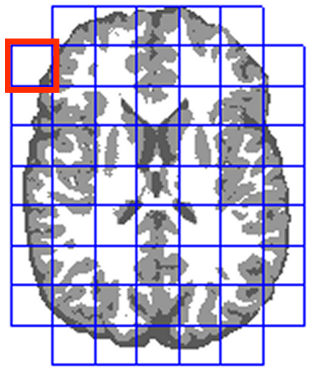

(b)

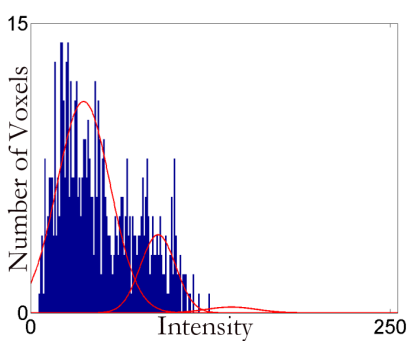

(c)

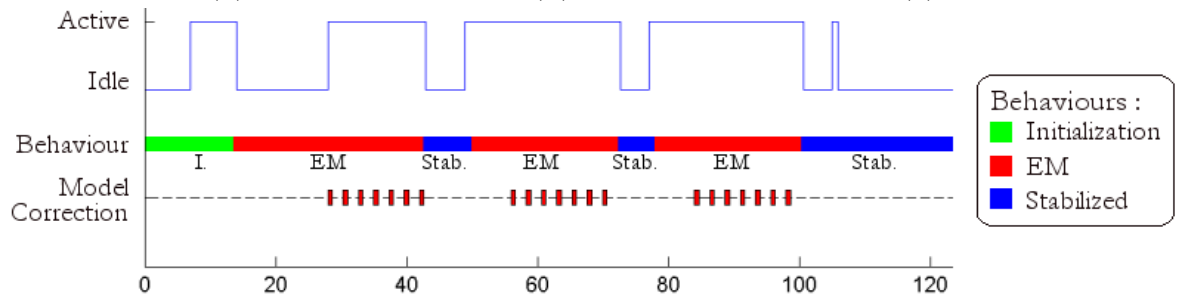

(d)

Fig. 6. Evaluation of tissue segmentation (b) on a real 3T brain scan (a). Our implementation allows to observe the activity of each agent: (c) shows the local histogram of the red-outlined agent in (b). All tissue classes are not represented, leading to bad models estimation without cooperation. Figure (d) shows the agent behaviour: after initialization it waits for the neighbouring agents to wake it up. Then it performs model corrections in its EM behaviour. After convergence (Stabilized behaviour), model checking restarts the estimation twice. Then, the agent stay in idle mode.

and cooperative agents which estimate local models. Coordination mechanisms are introduced (1) to ensure the spreading of knowledge between neighbouring agents and (2) to prevent agents from starting with poor knowledge. The main advantage over other existing local approaches [5],[6],[7] is that using this appropriate framework we are able to make full use of local estimation. In addition, it allows to integrate a priori information in an elegant way. Indeed, we can consider two levels of knowledge as regards MRI brain scans: the tissue knowledge at a local level and the brain structure knowledge at a global level. In general these two levels are processed independantly [9],[10]. We rather consider that they are linked and must be used in a common setting. We show how to introduce a priori anatomical knowledge expressed by fuzzy spatial relations in the MRF framework to segment several subcortical structures. We also show how to combine the MRF models of tissues and structures. Models are mutually constrained in their convergence, making both models gradually more accurate and providing optimal results for both levels. We thus show how the Markovian approach can be extended by introducing mechanisms (1) to handle multiple levels of regularizations (multi-scale regularization) and (2) to consider conjointly 
multiple level of heterogeneous knowledge (multi-scale knowledge processing). Our implementation provides interesting visualization tools which allow to track specific agents and explore local knowledge such as local segmentation errors or local intensity models. The evaluation shows competitive results compared to other algorithms with lower computational time. Indeed, local easy-to-segment territories converge quickly, allowing the system to focus on other areas. In addition, it appears to be robust to the intensity nonuniformity without any bias field assumption and estimation. Note that we currently consider a regular cubic partitioning for tissue segmentation but should in future work evaluate the contribution of more particular partitionning, like adaptive or spherical partitionings. The locality may allow to integrate brain extraction as an additional level of knowledge in our processing. We also plan to extend our model to new structures to study specific pathologies. Finally, MRF agent based computing appears as an interesting and modular tool for complex image segmentation.

\section{References}

1. Van Leemput, K., Maes, F., Vandermeulen, D., Suetens, P.: Automated modelbased tissue classification of MR images of the brain. IEEE Transaction on Medical Imaging 18(10) (1999) 897-908

2. Zhang, Y., Brady, M., Smith, S.: Segmentation of brain MR images through a hidden Markov random field model and the expectation-maximisation algorithm. IEEE Transaction on Medical Imaging 20(1) (2001) 45-47

3. Wells, W.M., Grimson, W.E.L., Kikinis, R., Jolesz, F.A.: Adaptative segmentation of MRI data. IEEE Transactions on Medical Imaging 15(4) (1996) 429-442

4. Ashburner, J., Friston, K.: Unified segmentation. NeuroImage 26 (2005) 839-851

5. Shattuck, D.W., Sandor-Leahy, S.R., Schaper, K.A., Rottenberg, D.A., Leahy, R.M.: Magnetic resonance image tissue classification using a partial volume model. NeuroImage 13(5) (2001) 856-876

6. Richard, N., Dojat, M., Garbay, C.: Automated segmentation of human brain MR images using a multi-agent approach. Artificial Intelligence in Medicine 30 (2004) $153-175$

7. Zhu, C., Jiang, T.: Multicontextual fuzzy clustering for separation of brain tissues in magnetic resonance images. NeuroImage 18(3) (2003) 685-96

8. Celeux, G., Forbes, F., Peyrard, N.: EM procedures using mean field-like approximations for model-based image segmentation. Pattern Recognition 36(1) (2003) $131-144$

9. Barra, V., Boire, J.: Automatic segmentation of subcortical brain structures in MR images using information fusion. IEEE Transaction on Medical Imaging 20(7) (2001) 549-558

10. Colliot, O., Bloch, I., Camara, O.: Description of brain internal structures by means of spatial relations for MR image segmentation. In: SPIE Medical Imaging. Volume 5370., San Diego (2004) 444-445

11. Collins, D., Zijdenbos, A., Kollokian, V., Sled, J., Kabani, N., Holmes, C., Evans, A.: Design and construction of a realistic digital brain phantom. IEEE Transactions on Medical Imaging 17(3) (1998) 463-468 